\title{
Torsional Vibration Mitigation by Harmonic Inversion through SPWM Carrier Signal Control
}

\author{
Antonino Di Gerlando, Khaled ElShawarby, Giovanni Maria Foglia, Roberto Perini
}

\begin{abstract}
The paper analyses torsional vibrations caused by electromagnetic torque harmonics in permanent magnet synchronous generators. A vibration mitigation method is proposed based on changing the phase angle of the carrier signal of sinusoidal PWM in order to invert the phase of the torque harmonic causing resonance. Since the frequency has not changed, the system is still in resonance. Thus, after a given time, the torque harmonic should be inverted again. The process is repeated as long as the system is at or near resonance. A criterion is presented to select the exact phase shift of the carrier needed in order to invert the torque harmonic. A method to calculate both the toggle time needed and the frequency range to apply such technique is presented. With the mitigation technique applied, two cases are considered on Matlab/Simulink, namely sustained operation at resonant frequency and accelerating the machine over some critical points.
\end{abstract}

Index Terms - Converter sequential command, PM synchronous machines, SPWM modulation, Torsional vibrations, vibration mitigation

\section{INTRODUCTION}

$\mathrm{T}$ ORSIONAL vibrations are a critical concern for any drive train. With no proper mitigation technique, vibrations can cause fatigue to the drive train in general. Excessive vibrations may break the shaft, since it is the weakest part in the drive train. In addition, even mild vibrations can cause an increase in the additional losses of synchronous machines, which depend on the vibration amplitude and frequency. Moreover, the thermal status can reach critical high levels because of vibrations [1].

Torsional vibrations can be given by electromagnetic causes such as variable frequency drives. Torque harmonics generated from the SPWM modulation may excite the natural frequencies of the drive train. Also non integer harmonics which have much lower amplitudes than the integer harmonics can be dangerous as they cover higher frequency range [2]. It has to be noted that vibrations may arise also because of other reasons, such as mechanical, due to unbalance of rotating parts; even the control closed loop interaction can amplify the vibrations [3] [4].

Due to their criticality, torsional vibrations have to be detected and mitigated.

There are various methods and equipments to measure or detect torsional vibrations, like accelerometers [5], PM rotary

Authors are with the Department of Energy at Politecnico di Milano, Via La Masa 34, 20156 Milano - Italy

e-mails: antonino.digerlando@polimi.it, khaled.elshawarby@polimi.it, gianmaria.foglia@polimi.it, roberto.perini@polimi.it acceleration sensors [6]. Oscillations can be also observed or detected, without direct mechanical measurement, from the motor speed, torque, flux or current [7], or from the error in the dc voltage or the generated power [2] [8].

A classical measure to reduce the torque harmonics amplitude is to shift harmonics to a higher order. It can be done by either increasing the number of pulses or the switching frequency of the converters or by increasing the number of output voltage levels [9][10]. However, such methods have some drawbacks like increasing the switching losses, the cost and complexity of the converter.

Based on the analysis done on SPWM in [11], a dependence of the phase of electromagnetic-torque harmonics on the phase of the carrier signal is here analyzed. Since resonance occurs when a harmonic torque component matches with the natural frequency of the system, the idea is to achieve an acceptable working condition at resonance through the phase angle control of the carrier signal.

In [15] the idea has been already proposed, however it lacks mathematical insight. Moreover, the proposed shifting angle was constant, independently of the harmonic order.

This paper deals with a standard radial flux permanent magnet synchronous generator (RF PMSG) directly driven (DD) by a wind turbine. The absence of a gear box is an advantage because it provides higher reliability and lower losses [12]. The generator voltage supplies a two-level converter, operating as rectifier, with a Sinusoidal PWM (SPWM) modulation. Torsional vibrations caused by airgap torque integer harmonics is the focus of the study. In section II, the machine data under study are reported, and the mechanical model of the system is presented. Section III reports the amplitude of the electromagnetic torque harmonics under SPWM modulation as a function of the phase angle applied to the carrier signal. The relation between the torque harmonics and the mechanical system is highlighted through the Campbell diagram. Section IV presents a vibrations mitigation method, and a criterion about how to choose the phase shift of the carrier signal is developed. In section $\mathrm{V}$, simulations were done on Matlab/Simulink for the system under study. Two cases are considered: steady-state operation at resonance and shaft acceleration through the resonant condition.

The effectiveness of the proposed technique is discussed.

\section{Two MASS SYSTEM MATHEMATICAL MODEL}

The system is a RF PMSG directly driven wind turbine (Fig.1a), whose data are presented in Table I.

The mechanical system is modelled by an equivalent lumped inertia model (two mass model), shown in Fig.1b: $J_{1}$, $\theta_{1}, J_{2}, \theta_{2}$ are the inertia and the deflection of the turbine and the rotor disk respectively, and $K$ is the shaft stiffness. 

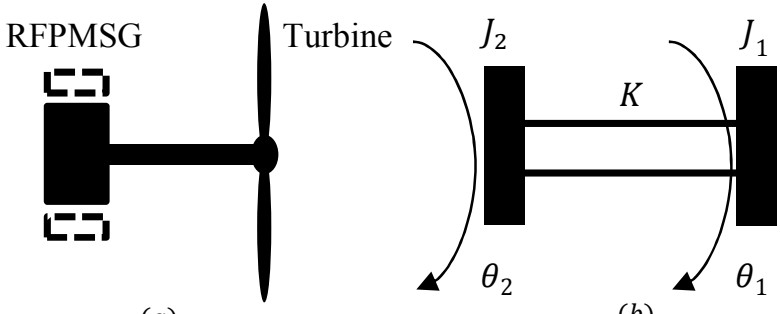

(b)

Fig. 1. a) actual system: a RF PMSG directly driven by a wind turbine; b) equivalent lumped inertia model.

TABLE I

RATED DATA OF ONE MW RF-PMSG MACHINE.

\begin{tabular}{l|l}
\hline$P_{n}[\mathrm{MW}], I_{n}[\mathrm{~A}], f_{n}[\mathrm{~Hz}]$ & $1.0 ; 713 ; 14.73$ \\
\hline Phase EMF at rated speed $E_{0}[\mathrm{~V}]$ (sinus. waveform) & 435 \\
\hline Rated torque $T_{n}[\mathrm{kNm}]$ & 561 \\
\hline Stator flux linkage due to PM, $\Psi_{P M}=E_{0} /\left(2 \pi \cdot f_{n}\right)[\mathrm{Wb}]$ & 8.147 \\
\hline No of poles for each rotor face $p, \Omega_{n}[\mathrm{rpm}]$ & 104,17 \\
\hline Phase resistance $R[\mathrm{~m} \Omega]$ & 14.59 \\
\hline Synchronous inductance $(\mathrm{d}, \mathrm{q}$ axes) $L[\mathrm{mH}]$ & 4.321 \\
\hline Rotor ext. diameter [mm], Stator ext. diameter $[\mathrm{mm}]$ & 5262,5586 \\
\hline Thickness of the hollow shaft [mm] & 450 \\
\hline Hollow shaft stiffness $K[\mathrm{Nm} / \mathrm{rad}]$ & $1.2 \cdot 10^{11}$ \\
\hline Axial length $[\mathrm{mm}]$ & 603 \\
\hline Turbine inertia $J_{1}$, Rotor inertia $J_{2}\left[\mathrm{~kg} \mathrm{~m}{ }^{2}\right]$ & $3 \cdot 10^{6}, 3.36 \cdot 10^{4}$ \\
\hline
\end{tabular}

The torsional natural frequency $T N F$ of a two-mass model is:

$$
T N F=\frac{1}{2 \pi} \sqrt{K / \frac{J_{1} * J_{2}}{\left(J_{1}+J_{2}\right)}} \quad[\mathrm{Hz}], \quad T N F=302.5 \mathrm{~Hz}
$$

\section{RELATION BETWEEN ELECTROMAGNETIC TORQUE AND RESONANT FREQUENCY.}

\section{A. Instantaneous Torque with SPWM Modulation}

The scheme of the drive is presented in Fig.2.

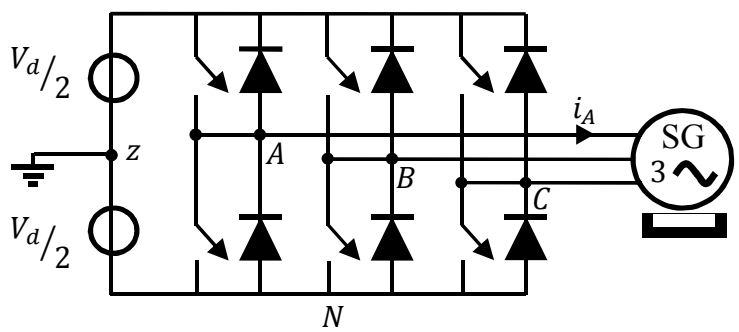

Fig. 2. Scheme of a PMSG connected to a two-level inverter. The dc bus voltage is assumed constant $\left(V_{d}\right)$; the load sign convention is used.

The machine can be modelled as a series $R$ - $L$-e circuit where $e(t)$ is sinusoidal. The two-level inverter is connected to the DC bus of voltage $V_{d}$. Point $z$ represents the virtual midpoint of the DC side capacitor.

The electromagnetic torque can be expressed as

$$
T_{\text {elm }}=(p / 2) \sqrt{3} \Psi_{P M} i_{q},
$$

where $\psi_{P M}$ is the RMS value of PM flux, $p$ is the number of poles, and $i_{q}$ is the quadrature current.

Due to (2), the harmonics present in the torque are the same as those in the current $i_{q}$.
By adopting the method developed in [11], the expression of $i_{q}$ is as follows:

$$
\begin{aligned}
& i_{q}(t)=\sqrt{3 / 2} I_{1} \sin (\varphi+\delta-\pi / 2) \\
& \quad+I_{m f-2} \sin \left(\left(m_{f}-3\right) \omega_{1} t-\delta+\psi_{c}-\vartheta_{m f-2}+\pi / 2\right) \\
& \quad-I_{m f-4} \sin \left(\left(m_{f}-3\right) \omega_{1} t+\delta+\psi_{c}-\vartheta_{m f-4}-\pi / 2\right) \\
& \quad-I_{m f+2} \sin \left(\left(m_{f}+3\right) \omega_{1} t+\delta+\psi_{c}-\vartheta_{m f+2}-\pi / 2\right) \\
& \quad+I_{m f+4} \sin \left(\left(m_{f}+3\right) \omega_{1} t-\delta+\psi_{c}-\vartheta_{m f+4}+\pi / 2\right) \\
& \quad-I_{2 m f-1} \sin \left(\left(2 m_{f}\right) \omega_{1} t+\delta+2 \psi_{c}-\vartheta_{2 m f-1}-\pi / 2\right) \\
& +I_{2 m f+1} \sin \left(\left(2 m_{f}\right) \omega_{1} t-\delta+2 \psi_{c}-\vartheta_{2 m f+1}+\pi / 2\right) \\
& +I_{3 m f-2} \sin \left(\left(3 m_{f}-3\right) \omega_{1} t-\delta+3 \psi_{c}-\vartheta_{3 m f-2}+\pi / 2\right) \\
& -I_{3 m f-4} \sin \left(\left(3 m_{f}-3\right) \omega_{1} t+\delta+3 \psi_{c}-\vartheta_{3 m f-4}-\pi / 2\right) \\
& -I_{3 m f+2} \sin \left(\left(3 m_{f}+3\right) \omega_{1} t+\delta+3 \psi_{c}-\vartheta_{3 m f+2}-\pi / 2\right) \\
& +I_{3 m f+4} \sin \left(\left(3 m_{f}+3\right) \omega_{1} t-\delta+3 \psi_{c}-\vartheta_{3 m f+4}+\pi / 2\right) \\
& \quad-I_{4 m f-1} \sin \left(\left(4 m_{f}\right) \omega_{1} t+\delta+4 \psi_{c}-\vartheta_{4 m f-1}-\pi / 2\right) \\
& +I_{4 m f+1} \sin \left(\left(4 m_{f}\right) \omega_{1} t-\delta+4 \psi_{c}-\vartheta_{4 m f+1}+\pi / 2\right)
\end{aligned}
$$

In (3), $I_{h}$ is the $h$-th harmonic amplitude, $\varphi$ the displacement between voltage and current fundamental components, $\delta$ the load angle, $m_{f}$ the SPWM frequency modulation ratio, $\omega_{1}$ the output fundamental angular frequency, $\psi_{c}$ the phase shift applied to the carrier signal. The current harmonic amplitudes and phases, $I_{h}$ and $\theta_{h}$, are obtained by applying each voltage harmonic, calculated in [11], to the equivalent $R-L-e$ circuit. The machine is vectorcontrolled to operate in maximum torque-per-ampere (MTPA) conditions; since the motoring convention is used, the internal emf has a phase displacement equal to $180 \mathrm{deg}$ with respect to the phase current.

Each group of terms of the same harmonic order is function of distinct multiple of $\psi_{c}$. Table II summarizes the harmonics present in the torque expression of (3).

TABLE II

HARMONIC CONTENT IN THE ELECTROMAGNETIC TOROUE RIPPLE AND, FOR Each Harmonic, Phase COMPONENT DUe TO THE ShIFT $\Psi_{C}$ OF THE CARRIER SIGNAL

\begin{tabular}{c|c}
\hline $\boldsymbol{h}$ & Phase component due to the shift $\boldsymbol{\psi}_{c}$ \\
\hline $\boldsymbol{m}_{\boldsymbol{f}} \pm \mathbf{3}$ & $\psi_{c}$ \\
\hline $\mathbf{2} \boldsymbol{m}_{\boldsymbol{f}}$ & $2 \psi_{c}$ \\
\hline $\mathbf{3} \boldsymbol{m}_{\boldsymbol{f}} \pm \mathbf{3}$ & $3 \psi_{c}$ \\
\hline $\mathbf{4} \boldsymbol{m}_{\boldsymbol{f}}$ & $4 \psi_{c}$ \\
\hline
\end{tabular}

By means of (3), the amplitude of any torque harmonic at any speed can be evaluated. The amplitudes of the main harmonics of Table II are shown in Fig. 4, as a function of the machine speed expressed as the frequency of the PWM control signals, [Hz]; they are reported in p.u., referred to the rated torque $T_{n}$. The chosen frequency ratio $m_{f}$ is as low as 15 in order to limit the switching losses. The power generation to the grid starts from the cut-in speed (normally $0.3-0.35 \cdot \Omega_{n}$ ) up to the rated one $\Omega_{n}$. 


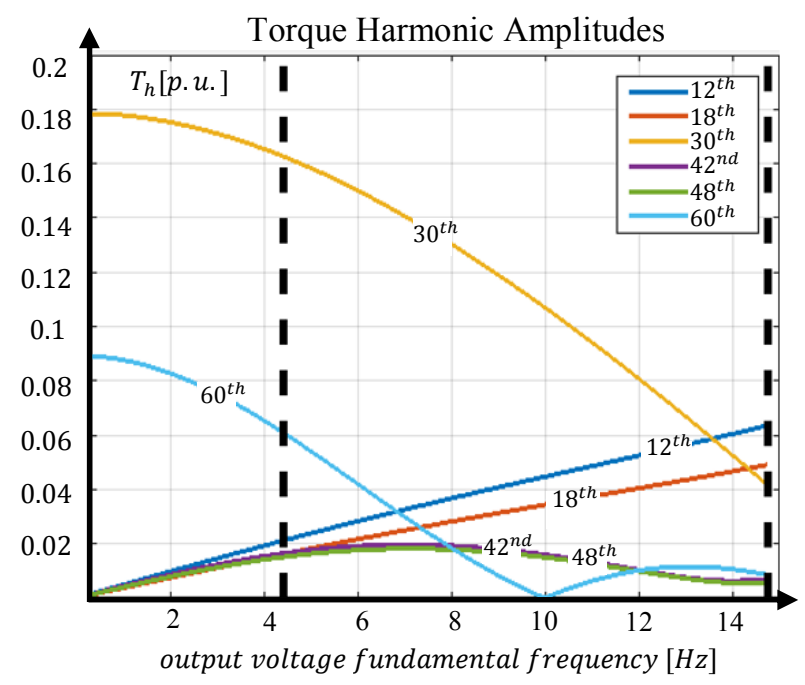

Fig. 4. Torque harmonic amplitudes, in p.u., of order $m_{f} \pm 3,2 m_{f}, 3 m_{f} \pm 3,4 m_{f}$ across the whole range of operation namely $(0.3 \sim 1) f_{n}$, represented by vertical lines. Frequency ratio $m_{f}=15$.

Some remarks should be highlighted:

- the machine is controlled through two cascaded PI controllers: an inner current-controlled loop and an outer speed-controlled one. Their cut-off angular frequencies are set to $25 \mathrm{rad} / \mathrm{s}$ and $2 \mathrm{rad} / \mathrm{s}$ respectively. Since the former is low, the current cannot follow the constant reference, and its waveform has all the high order harmonics;

- the harmonic of the order $30^{\text {th }}\left(2 \cdot m_{f}\right)$ is the highest in amplitude; while harmonic of order $60^{\text {th }}$ reaches zero at a point. This can be explained by the Bessel functions used in the calculations of the voltage harmonic amplitudes in [11].

\section{B. Campbell Diagram}

The previous sections have identified both the natural frequency of the system and the torque amplitudes for all the dominant harmonics in p.u.

The Campbell diagram describes the relation between the mechanical and electrical interactions (Fig.5). The machine speed is plotted on the $\mathrm{x}$-axis as the frequency of the PWM control signals, the torque harmonic frequencies are on the $y-$ axis. The torsional natural frequency (TNF) is constant and plotted parallel to the $\mathrm{x}$-axis. The intersections between the electrical frequencies (torque harmonics) and the $T N F$ are called critical points which resemble the occurrences of torsional resonance and can be found by:

$$
f_{c r}=\frac{T N F}{h}
$$

The following has to be taken into account:

- from a theoretical viewpoint, the resonance involves just a frequency value; in practice, the resonance danger involves a frequency range around the theoretical value namely the beat phenomenon: thus, it is more reasonable to consider not only the critical point, but a critical band instead;

- another key factor is the amplitude of the exciting harmonic: a harmonic of an amplitude as small as $1 \%$ of the rated torque can be dangerous during resonance [13]; however, larger harmonic torque amplitudes accelerate the build-up of vibrations.
Table III displays the critical frequencies with their corresponding harmonic amplitudes. It can be noted that the highest torque amplitude is by harmonic $2 \cdot m_{f}=30$.

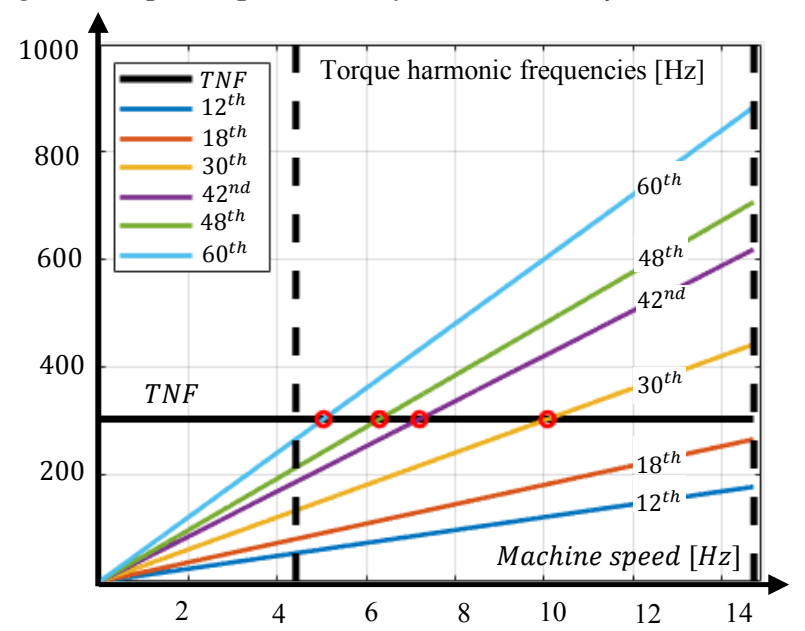

Fig. 5. Campbell diagram of the RF PMSG under SPWM modulation with a frequency ratio $m_{f}=15$. The $T N F$ is $302.5 \mathrm{~Hz}$ and the vertical lines represent the range of operation from $(0.3 \sim 1)$ of the rated speed $\Omega_{n}$.

TABLE III

TORQUE HARMONIC AMPLITUDES OF THE CRITICAL POINTS OF THE MECHANICAL SySTEM (RED CiRCLED PoinTs IN FIG. 5)

\begin{tabular}{c|c|c|c|c}
\hline$f_{c r}[\mathrm{~Hz}]$ & $f_{c r} / f_{n}[\%]$ & $\begin{array}{c}\text { Excited } \\
\text { Harmonic }\end{array}$ & $\begin{array}{c}T_{h} / T_{r}[\%] \\
T_{h} \text { Amplitude } \\
{[\mathrm{kNm}]}\end{array}$ \\
\hline 5.04 & 34.2 & 60 & $5.36 \%$ & 30.1 \\
\hline 6.30 & 42.8 & 48 & $1.79 \%$ & 10 \\
\hline 7.20 & 48.9 & 42 & $1.97 \%$ & 11.1 \\
\hline 10.1 & 68.6 & 30 & $10.6 \%$ & 59.5 \\
\hline
\end{tabular}

\section{Mitigation METHOD: DESCRIPTION AND ANALYSIS}

As observed in Section III, the phase of torque harmonics is a function of the shift angle imposed to triangular carrier signal $\psi_{c}$. Thus by changing $\psi_{c}$, the phase of these harmonics can be changed. The proposal is that, during resonance, the torque harmonic exciting the natural frequency be inverted, by an appropriate phase shift applied to the carrier signal. Inverting the harmonic will cause the angular deflection to transiently decrease and then start growing again, as the system is still at resonance.. Therefore, the phase of the torque harmonic has to be reverted again, for example every time interval $t_{\text {toggle }}$; this process is to be repeated as long as the system is near or at resonance. Fig.6 illustrates the proposal. Some aspects should be considered:

a) inversion of the torque harmonic;

b) suitable value of $t_{\text {toggle }}$;

c) critical frequency range;

d) application in a three-phase system.

\section{A. Inversion of the Torque Harmonic.}

Recalling Table II, the phase of each torque harmonic component includes a distinctive multiple of $\psi_{c}$. The goal is to impose a $180^{\circ}$ phase shift, after each $t_{\text {toggle }}$. For example, considering the first critical point, the $60^{\text {th }}$ harmonic is the one exciting the $T N F$.

For $h=4 \cdot m_{f}=60$, the phase in Table II includes the quantity $4 \cdot \psi_{c}$; thus:

$$
4 \cdot \psi_{c}=180^{\circ}+k 360^{\circ} \quad \therefore \psi_{c}=45^{\circ}+k 90^{\circ}
$$


(a)

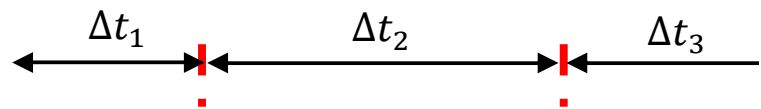

(b)

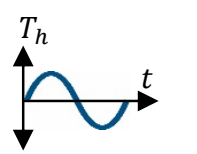

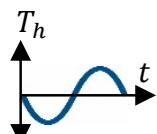

(c)

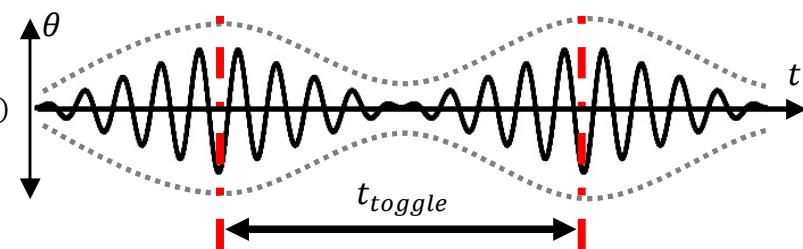

Fig. 6. Mitigation method during resonance at $f=f_{c r}$, changing the phase of exciting harmonic with $180^{\circ}$ after a time $t_{\text {toggle }}$. (a): subsequent time intervals during the resonance. (b): phase of the harmonic $T_{h}$ which is exciting the resonance, in any interval. (c): resultant behavior of the angular deflection $\theta$.

In order to invert the $60^{\text {th }}$ torque harmonic, the phase $\psi_{c}$ of the carrier signal should toggle between two values: 0 and $45^{0}$ (or $135^{0}, 225^{0} \ldots$ ).

Table IV shows the critical points and the necessary shift angle to the carrier signal in order to achieve the inversion of torque harmonic.

TABLE IV

VALUES OF THE SHIFT $\Psi_{C}$ OF THE TRIANGULAR SignAL USED TO INVERT THE HARMONIC EXCITING THE TNF

\begin{tabular}{c|c|c|c}
\hline$f_{c r}[\mathrm{~Hz}]$ & $f_{c r} /_{f_{n}}[\%]$ & $\begin{array}{c}\text { Excited } \\
\text { Harmonic }\end{array}$ & $\Psi_{c}$ \\
\hline$f_{c r 1}=5.04$ & 34.2 & 60 & $45^{0},\left(135^{0}, 225^{0} \ldots\right)$ \\
\hline$f_{c r 2}=6.30$ & 42.8 & 48 & $60^{0},\left(180^{0}, 300^{0} \ldots\right)$ \\
\hline$f_{c r 3}=7.20$ & 48.9 & 42 & $60^{0},\left(180^{0}, 300^{0} \ldots\right)$ \\
\hline$f_{c r 4}=10.1$ & 68.6 & 30 & $90^{0},\left(270^{0}, 450^{0} \ldots\right)$ \\
\hline
\end{tabular}

\section{B. Selection of Toggle Time $t_{\text {toggle. }}$}

From the mathematical viewpoint, the inversion, achieved by imposing a phase shift to the carrier signal, modifies the harmonic spectrum: the targeted harmonic $h_{x}$, the one causing the $T N F$ excitation, is removed and other harmonics are generated. In general, the new generated harmonic amplitudes are lower than the original one $h_{x}$ to be removed; moreover, the harmonic amplitudes depend on the order $h_{x}$.

In order to understand how the new spectrum is formed, consider a sample even harmonic of amplitude 1 and order $h_{x}$. First, let it be inverted at half of the observation period $\tau$, i.e. $t_{\text {toggle }}=\tau / 2$ (Fig. 7a). The result waveform is an even function with quarter wave symmetry: thus, the Fourier series expansion-only contains odd harmonics. The coefficient amplitudes are:

$$
\begin{gathered}
F_{\frac{1}{2}}=\frac{1}{\pi} \int_{0}^{2 \pi} f(\theta) \cos (h \theta) d \theta=\frac{4}{\pi} \int_{0}^{\frac{\pi}{2}} \sin \left(h_{x} \theta\right) \cos (h \theta) d \theta \\
\cdot F_{\frac{1}{2}}=\frac{4}{\pi}\left[\frac{h_{x}}{h_{x}^{2}-h^{2}}\right] \quad h_{x}, h \in N \quad h: \text { odd; } h_{x}: \text { even }
\end{gathered}
$$

Considering the inversion every $1 / 4 \tau\left(t_{\text {toggle }}=\tau / 4\right)$, the result is an even function and the Fourier coefficients equal:

$$
F_{\frac{1}{4}}=\frac{2}{\pi}\left[\int_{0}^{\frac{\pi}{2}} \sin \left(h_{x} \theta\right) \cos (h \theta) d \theta-\int_{\frac{\pi}{2}}^{\pi} \sin \left(h_{x} \theta\right) \cos (h \theta) d \theta\right]
$$

$$
\begin{aligned}
& \therefore \quad F_{\frac{1}{4}}=\frac{1-2 \cos \left(\left(h_{x}+h\right) \frac{\pi}{2}\right)+\cos \left(\left(h_{x}+h\right) \pi\right)}{\pi \cdot\left(h_{x}+h\right)}+ \\
& \& \quad F_{\frac{1}{4}}=\frac{1}{\pi h_{x}}\left[1-\cos \left(h_{x} \pi\right)\right]=0 \quad h=h_{x} \& h_{x} \text { :even }
\end{aligned}
$$

As an example, consider the harmonic $h_{x}=12$ in Fig.7a: $t_{\text {toggle }}=\tau / 2, \tau / 4, \tau / 6$ in Fig. $7 \mathrm{~b}, \mathrm{c}, \mathrm{d}$ respectively. Based on (6) and (8), the corresponding non-null harmonics are given in the tables.
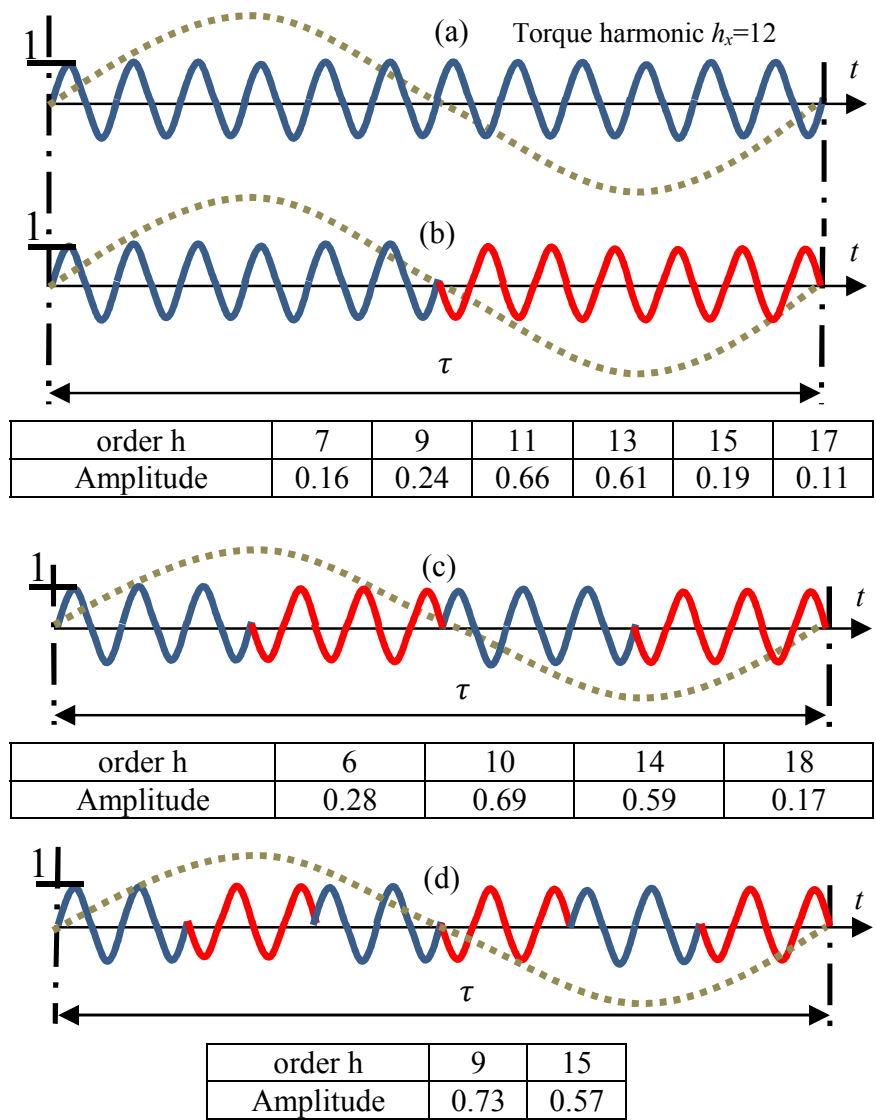

Fig.7. Torque harmonic inversion examples (with $h_{x}=12$ ), and modified spectrum: a) reference condition; b) inversion at half observation period $\tau$ : $t_{\text {toggle }}=\tau / 2$; c) $t_{\text {toggle }}=\tau / 4 ;$ d) $t_{\text {toggle }}=\tau / 6$.

The new spectrum can be interpreted in the following way: the inversion at $\tau / 2$ shifts the targeted harmonic by one position in each direction, the inversion at $\tau / 4$ shifts the targeted harmonic by two positions in each direction and the inversion at $\tau / 6$ shifts the targeted harmonic by three positions. So more in general, for a generic integer $a$, a correlation can be found between the toggle time $\tau / a$ and the number of shifting positions $p_{x}$ :

$$
p_{x}=a / 2 \text { for } t_{\text {toggle }}=\tau / a
$$

\section{Critical Frequency Range.}

As explained earlier, the technique modifies the harmonic spectrum in such a way to eliminate a targeted harmonic which is suitable for operation at a fixed speed near or at resonance speed $f_{c r}$. However, the proposed technique 
generates other harmonics creating other critical points. This has to be taken into account for the application of the method during the machine acceleration passing a selected critical frequency. In other words, during machine acceleration, if the harmonic inversion technique is not applied, resonance occurs at the critical frequency $f_{c r}$. However, if the proposed technique is applied indefinitely, resonance will occur at other critical frequencies, corresponding to the new generated harmonics.

Thus a suited frequency range is to be chosen for the application of the inversion technique. A suitable value for the safest frequency range $f_{a p p l}$ to apply the inversion method is:

$$
\frac{h_{x}}{h_{x}+p_{x} / 2} \cdot f_{c r} \leq f_{a p p l} \leq \frac{h_{x}}{h_{x}-p_{x} / 2} \cdot f_{c r}
$$

In order to better clarify the point, consider the torque harmonic $h_{x}=2 \cdot m_{f}=30 ; t_{\text {toggle }}$ equals one quarter of the control signal period $T_{s} / 4$; the machine is supposed to accelerate from 0.61 p.u. to 0.81 p.u. (i.e. $9 \mathrm{~Hz}$ to $12 \mathrm{~Hz}$ ). The new torque harmonic spectrum can be calculated with (8) (Fig.8a).

The new critical speeds, i.e. control signal frequencies, are 9.45, $10.8 \mathrm{~Hz}$ instead of $f_{c r 4}=10.1 \mathrm{~Hz}$ (found from the intersection of the TNF with the new harmonic lines in the Campbell diagram, Fig.8b). This means that, without the mitigation technique, a resonance condition will occur at $f_{c r 4}=10.1 \mathrm{~Hz}$. However, if the mitigation technique is applied indefinitely, resonance will occur twice, at 9.45 and $10.8 \mathrm{~Hz}$.

Therefore, in order to avoid all resonance conditions, the mitigation method has to be applied in an intermediate range, from $9.76 \mathrm{~Hz}$ to $10.4 \mathrm{~Hz}$ (through (11)), presented in the shaded part in Fig. 8 b.
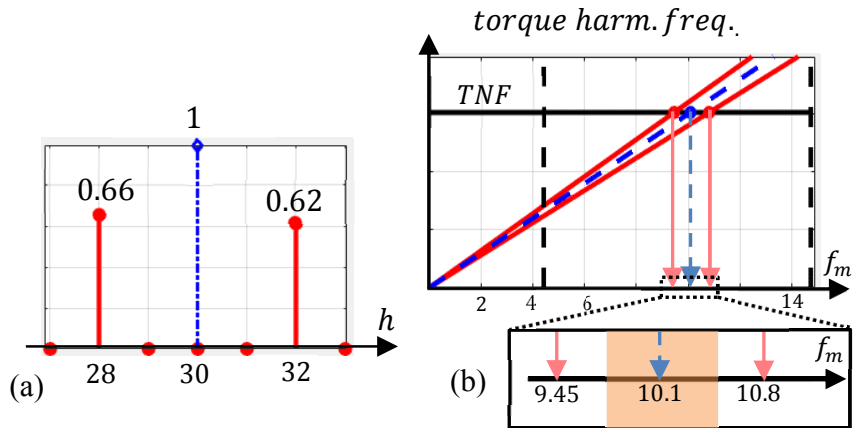

Fig. 8. a) Torque harmonic spectrum showing the targeted harmonic to be eliminated ( $h_{x}=30$, of amplitude 1), and the new generated harmonics;

(b) Corresponding Campbell diagram. The shaded part is the proposed frequency range to which apply the mitigation technique.

\section{D.Application to a Three-Phase System.}

The effect of a shifted triangular carrier waveform in a three-phase modulation (three sinusoidal control signals) is peculiar and needs a dedicated analysis to be undertaken with the help of Fig.9b, where an inverted carrier signal is introduced at $0.5 \cdot T_{s}$ at rated frequency (i.e. $T_{s}=68 \mathrm{~ms}$ ). The analysis is done based on the derivation in [14] for the expression of the output voltages $v_{A N}, v_{B N}, v_{C N}$. The original harmonic content of normal SPWM follows $h=m \cdot m_{f}+n$ for $m_{f}=3 \cdot k(k, n, m \in \mathrm{N})$ (Fig.9a).

Focusing on $m=3$ and $m_{f}=15$ we get the voltage and current harmonics $(45,(43,47),(41,49)$.. $)$. The torque components corresponding to these groups of harmonics are the $42^{\text {nd }}$ and the $48^{\text {th }}$ since:

- the $45^{\text {th }}$ current harmonic is a zero sequence component [11];

- $41^{\text {st }}, 43^{\text {rd }}$ current harmonics contribute in the generation of the $42^{\text {nd }}$ torque harmonic;

- $47^{\text {th }}, 49^{\text {th }}$ current harmonics contribute in the generation of the $48^{\text {th }}$ torque harmonic.

As shown in Table IV, both torque harmonics are of concern to the torsional vibrations and can be inverted by $\psi_{c}$ equal to $180^{\circ}$. As explained in sSection IV.B, having inverted the triangular at half of the control signal period $T_{s}$, the voltage and current harmonics of concern (namely $41^{\text {st }}$, $43^{\text {rd }}, 47^{\text {th }}, 49^{\text {th }}$ ) are eliminated. Thus the new harmonics generated are shifted by one position, and they are $40^{\text {th }}, 42^{\text {nd }}$, $44^{\text {th }}, 48^{\text {th }}, 50^{\text {th }}$ (Fig. 9b).

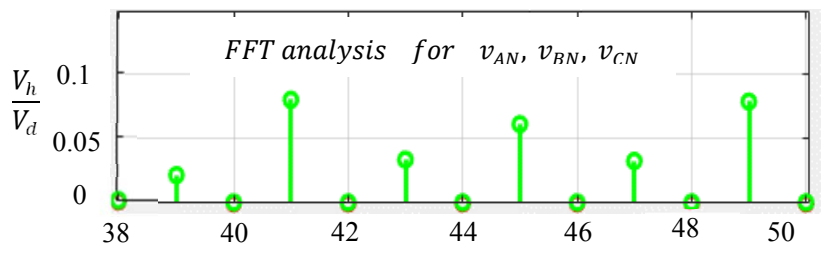

(a)
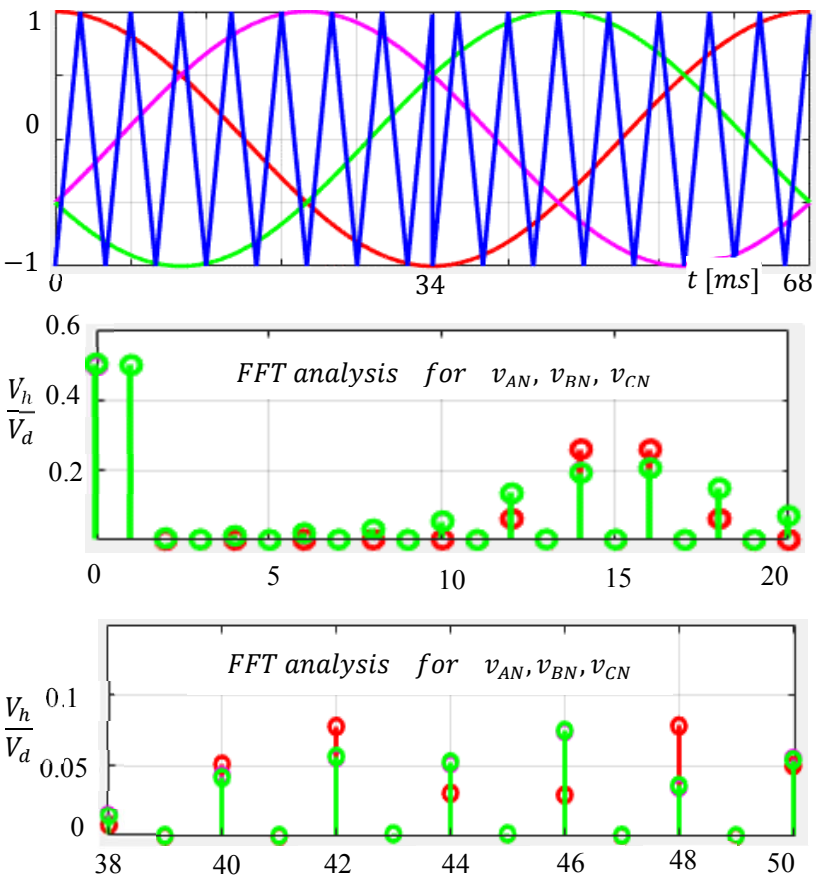

(b)

Fig. 9. a) FFT analysis of the voltages $v_{A N}, v_{B N}, v_{C N}$ for typical SPWM for $m_{f}=15$. All harmonic amplitudes are overlapping for all three phases. b) Proposed carrier signal inverted at half of the control signal period and FFT analysis for the corresponding voltages $v_{A N}$ (in red), $v_{B N}, v_{C N}$ (overlapping in green color). Asymmetry exists, since no overlap for harmonics of all three phases occurs.

Two remarks on the voltage FFT analysis (Fig.9b) hold:

- low order harmonics are present in the voltages;

- an asymmetry arises, since harmonic amplitudes of three voltages are not overlapping.

In order to overcome the asymmetry, a solution is adopting a $t_{\text {toggle }}$ as a multiple of $1 / 3$ of the control signal period $T_{s}$ : in this way, the inversion instant is the same for all the three 
phases. Fig.10 displays the proposed inversion technique every $1 / 3 \cdot T_{s}$.

The following remarks can be made:

- such a fraction of (1/3) is required in order to avoid asymmetry among the phases;

- the proposed inversion of the carrier signal with the $1 / 3 T_{s}$ fraction generates interharmonics;

- low order harmonics are much reduced compared to the inversion at $0.5 T_{s}$

In [15], only a 180 degree shift for the carrier signal was proposed, but this is not always appropriate: as shown in Table IV, each harmonic requires a different $\psi_{c}$ value. Moreover, in [15] no criterion to select $t_{\text {toggle }}$ or $f_{\text {appl }}$ was defined.
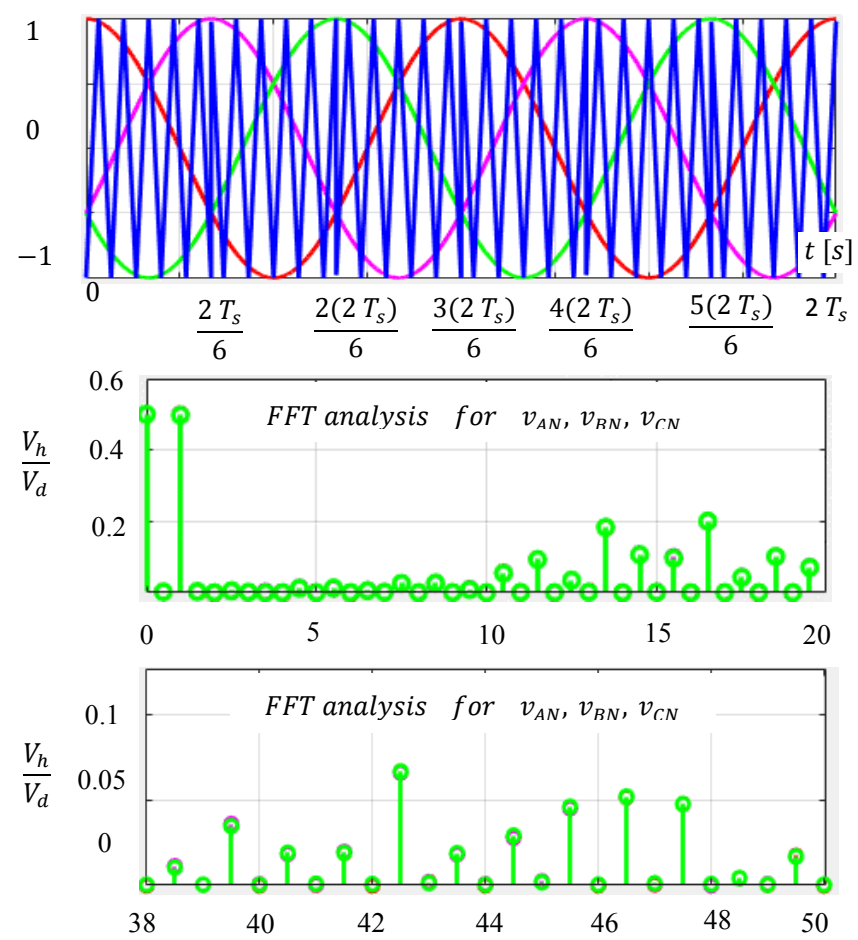

Fig. 10. Proposed carrier signal inverted at $1 / 3$ of the control signal period $T$ $(68 \mathrm{~ms})$, and FFT analysis for the corresponding voltages $v_{A N}, v_{B N}, v_{C N}$ Overlap of harmonics of all three phases occurs, due to the 3 phase voltage equality.

\section{SimUlation RESUlts AND DisCUSSION}

A Matlab/Simulink model is used as the simulation basis. Each machine module is modelled by the standard two-axis (dq) model of the PM brushless machine. For each module, the classical field oriented control is implemented, aimed at MTPA condition. Two cascaded controllers are implemented: current (internal) and speed (external) controllers; their bandpasses equal $40 \mathrm{rad} / \mathrm{s}$ and $5 \mathrm{rad} / \mathrm{s}$ respectively.

In order to test the effectiveness of the proposed technique, two cases are considered. The first is the operation at the critical point with the highest amplitude selected (i.e. $f_{c r} 4$ $=10.1 \mathrm{~Hz}$ ). During normal operation, the deflection reaches its maximum permissible limit in less than one minute, i.e. the shaft breaks. In such a critical point the harmonic exciting the $T N F$ is the $30^{\text {th }}$ (Table II). In order to invert the phase of the torque harmonic, $\psi_{c}=90 \mathrm{deg}$ is considered, and $t_{\text {toggle }}=2 / 3 \cdot T_{s}$ is adopted. The effect on torque waveform is presented in Fig.11. During resonance, the angular deflection is between fixed envelope thanks to the mitigation method proposed.

The second case, presented in Fig.12, is to accelerate the machine across the first critical point in which the $60^{\text {th }}$ torque harmonic excites the natural frequency. A toggle time of $T_{s} / 6$ is adopted and $\psi_{\mathrm{c}}=45^{\circ}$. The mitigation method is applied over the speed interval $4.92 \sim 5.17 \mathrm{~Hz}$, calculated from (11). It could be noted that the maximum deflection permissible is:

$$
\theta_{\text {max }}=\frac{0.8 * \sigma_{\max }}{G} \frac{l_{\text {sh }}}{R_{\text {ext }}} \cong 4.10^{-4} \mathrm{rad}
$$

with $\sigma_{\max }$ maximum stress allowable for the material (117.7 $\mathrm{MPa}$ for steel), $G$ shaft shear modulus ( $79.3 \mathrm{GPa}$ for steel), $l_{\text {sh }}$ shaft length, $R_{\text {ext }}$ shaft external radius.

Outside such an interval, normal SPWM is applied. As it can be seen, at resonant frequency, the angular deflection is limited below $\hat{\theta}=0.06 \cdot 10^{-4} \mathrm{rad}$, less then $\theta_{\max }$. In addition, the increasing-decreasing behaviour of the deflection angle due to the change of the carrier signal shift angle can be observed.

Table $\mathrm{V}$ shows the critical points and the corresponding frequency range where to apply the change of the carrier signals. Outside these ranges normal SPWM should be applied. It also reports the absolute maximum angular deflection of the rotor under resonance with the proposed mitigation strategy. In all cases the highest deflection is less than $\hat{\theta}=0.05 \cdot 10^{-4} \mathrm{rad}$, less then $\theta_{\max }$.

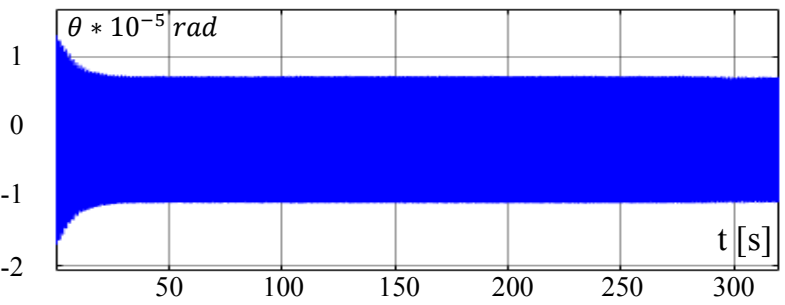

(a)

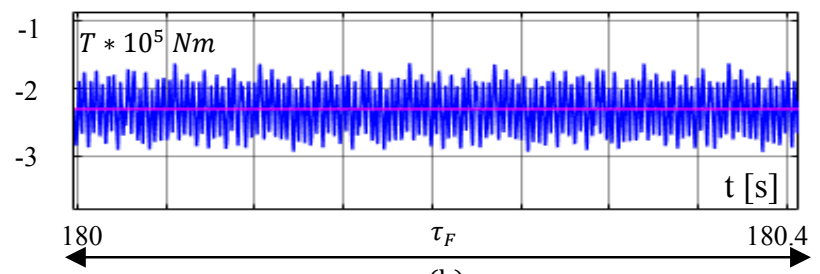

(b)

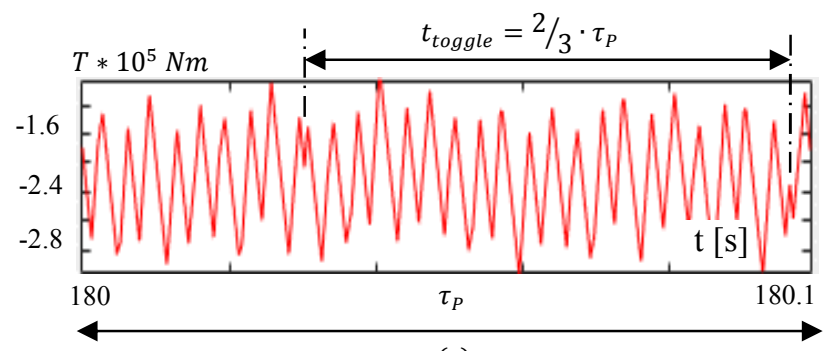

(c)

Fig. 11. Waveforms of the PMSG rotor at $f_{c r 4}=10.1 \mathrm{~Hz}\left(T_{s}=0.1 \mathrm{~s}\right)$, when the proposed technique of carrier inversion is applied every $2 / 3 \cdot T_{s}$. The fundamental period $\tau_{F}=4 * T_{s}=0.4 \mathrm{~s}$ while the principle one $\tau_{P}=T_{s}=0.1 \mathrm{~s} \mathrm{.} \mathrm{a)}$ angular deflection, along a $320 \mathrm{~s}$ interval; b) electromagnetic torque waveform vs. the torque reference, over the fundamental period $\tau_{F}$. c) electromagnetic torque waveform over the principle period $\tau_{P}$ under the carrier inversion applied every $t_{\text {toggle }}=2 / 3 \cdot T_{s}$. 


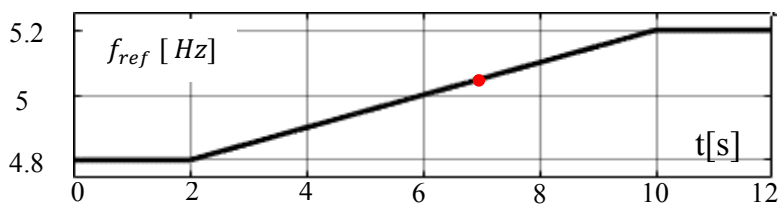

(a)

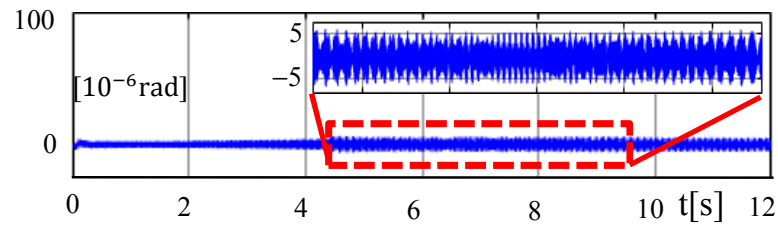

(b)

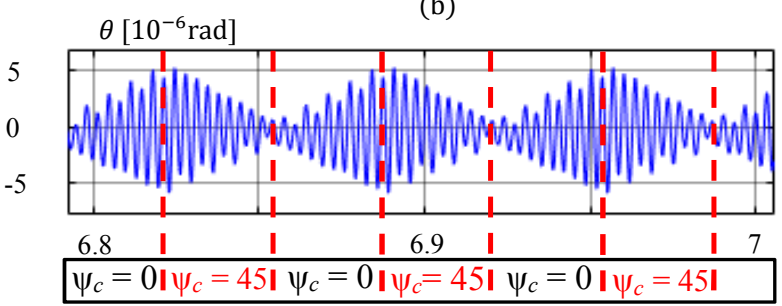

(c)

Fig. 12. Operation under acceleration through the critical frequency $f_{c r 1}$ excited by the harmonic of order $60^{\text {th }}$ with the carrier shift every $1 / 6 \cdot T_{s}:$ a) frequency ramp b) angular deflection; the red rectangle highlights the frequency range where the mitigation method is applied and also shows that the deflection is limited between $\pm 5 \cdot 10^{-6}$. c) shows the carrier shift instants and their effect on the angular deflection.

TABLE V

SIMULATION DATA FOR ALL THE CRITICAL POINTS

\begin{tabular}{c|c|c}
\hline$f_{c r}[\mathrm{~Hz}]$ & $\begin{array}{c}\text { Frequency Range } \\
{[\mathrm{Hz}]}\end{array}$ & $\begin{array}{c}|\hat{\theta}| \text { max deflection angle } \\
{\left[10^{-4} \mathrm{rad}\right]}\end{array}$ \\
\hline$f_{c r 1}=5.04$ & $4.92 \sim 5.17$ & $\leq 0.05$ \\
\hline$f_{c r 2}=6.30$ & $6.11 \sim 6.50$ & $\leq 0.02$ \\
\hline$f_{c r 3}=7.20$ & $6.96 \sim 7.46$ & $\leq 0.008$ \\
\hline$f_{c r 4}=10.1$ & $9.60 \sim 10.61$ & $\leq 0.06$ \\
\hline
\end{tabular}

\section{CONCLUSION}

The paper considered the problem of torsional vibrations in a PMSG directly driven by a wind turbine. A technique to mitigate torsional vibrations through the control of the phase of the carrier signal has been introduced. An electrical analysis has been done to estimate the torque harmonics amplitude.

A Campbell diagram presented the critical points where resonance may occur. A criterion was defined to select the correct angle to apply for the carrier signal in order to invert the torque harmonics causing resonance. It is a repetitive process applied every given time called $t_{\text {toggle }}$. It should be applied as long as the system is near or at resonance, which is given as a frequency range $f_{\text {appl }}$. A method about how to select both $t_{\text {toggle }}$ and $f_{\text {appl }}$ has been proposed.

The system has been simulated by Matlab/Simulink, and the soundness of the proposed technique has been proved. Two cases were considered: operation at resonance frequency and acceleration through all critical points. Angular deflection is much reduced by applying the proposed technique.
Future activity will concern experimental tests, also investigating how to detect resonance occurrence.

\section{REFERENCES}

[1] A. Arkkio, S. Cederstrom, H. A. Awan, S. Saarakkala, T. Holpainen, "Additional Losses of Electrical Machines under Torsional Vibration" IEEE Trans. on Energy Conversion, Vol. PP, Issue 99, 2017

[2] H. Geng, D. Xu, B. Wu, G. Yang, "Active Damping for PMSG-Based WECS With DC-Link Current Estimation" IEEE Trans. on Ind. Electr., Vol. 58, Issue 4, 2011, pp.1110-1119

[3] J.S. Manguelle, S. Schroder, T. Geyer, G. Ekemb, J. Nyobe-Yome, "Prediction of Mechanical Shaft Failures Due to Pulsating Torques of V-F Drives” IEEE Trans. Ind. Appl., V. 45, No 5, 2010, pp.1979-1988.

[4] M. Mauri, M. Rossi, M. Bruha, "Generation of Torsional Excitation in a Variable-Speed-Drive System" Int. Symp. on Power Electronics, Electrical Drives, Automation and Motion (SPEEDAM), 2016

[5] S. Schramm, C. Sihler, J. S. Manguelle, P. Rotondo, "Damping Torsional Interharmonic Effects of Large Drives" IEEE Trans. on Power Electronics, Vol. 25, Issue 4, 2010, pp.1090-1098

[6] J. Zhang, H. Feng, "The permanent magnet rotating (angular) acceleration sensor and a new method of mechanical rotating system torsional vibration measuring” Contr. \& Decision Conf. (CCDC), 2017

[7] M. Bruha, M. Byrtus, K. Pietilainen, M. Rossi, M. Mauri, "Torsional Issues Related to Variable Frequency Control of Elastic Drive Systems" 42 ${ }^{\text {nd }}$ IEEE Ann. Conf. on Ind. El. Society (IECON), 2016

[8] H. Geng, D. Xi, B. Wu, G. Yang, "Active Damping for Torsional Vibrations in PMSG based WECS" $25^{\text {th }}$ Annual IEEE Applied Power Electronics Conference and Exposition (APEC), 2010

[9] M. Bruha, Z. Peroutka, "Torsional Vibration in Large Speed Drive Systems: Origin and Mitigation Methods" $17^{\text {th }}$ European Conference on Power Electronics and Applications (EPE'15 ECCE-Europe), 2015

[10] S. Schramm, C. Sihler, J. S. Manguelle, P. Rotondo, "Damping Torsional Interharmonic Effects of Large Drives" IEEE Trans. on Power Electronics, Vol. 25, Issue 4, 2010, pp.1090-1098

[11] A. Di Gerlando; K. ElShawarby; M. Iacchetti, G. M. Foglia; R. Perini: "DC Current and Torque Ripple Mitigation in Modular PMSGs Drives for Multi-MW WECS with Linear PWM Inverter Modulation," submitted to XXIII Int. Conf. on Electrical Machines (ICEM), 2018.

[12] H. Polinder, F. van der Pijl, G. de Vilder, P. Tavner, "Comparison of Direct-Drive and Geared Generator Concepts for Wind Turbines" IEEE Trans. on En. Conv., Vol. 21, Issue 3, 2006, pp.725-733.

[13] J. S. Manguelle, C. Sihler, J. Nyobe-Yome, "Modelling of Torsional Resonances for Multi-Megawatt Drives Design" IEEE Industry Applications Society Annual Meeting (IAS), 2008

[14] D.G. Holmes, B. P. McGrath, "Opportunities for Harmonic Cancellation with Carrier Based PWM for Two-Level and Multi-Level Cascaded Inverters", Ind. Appl. Conf., 1999, pp. 781-788 vol.2

[15] J. Manguelle, C. Sihler, S. Schramm, "A General Approach of Damping Torsional Resonance Modes in Multimegawatt Applications" IEEE Trans. on Ind. Appl., Vol.47, Issue 3, 2011, pp.1390-1399

\section{VIII.BIOGRAPHIES}

Antonino Di Gerlando received his MS degree in electrical engineering from the Politecnico di Milano, Italy, in 1981. Currently, he is a Full Professor at the Department of Energy at Politecnico di Milano. Fields of interest: design and modeling of electrical machines, converters and drive systems. He is a senior member of IEEE, and member of the Italian Association AEIT.

Khaled Elshawarby received his MS degree in electrical engineering at Politecnico di Milano, Milano, Italy 2016. Currently, he is a PhD student in Electrical Engineering at Politecnico di Milano, Italy. His interests are in power electronics and electrical machines.

Giovanni Maria Foglia received his MS degree and the $\mathrm{PhD}$ in electrical engineering at Politecnico di Milano, Milano, Italy, in 1997 and 2000.

Currently, he is an Assistant Professor at the Department of Energy at Politecnico di Milano, and his main field of interest is the analysis and design of PM electrical machines.

Roberto Perini (M'10) received his MS degree and the $\mathrm{PhD}$ in electrical engineering from the Politecnico di Milano, Milano, Italy.

Currently, he is an Associate Professor at the Department of Energy at Politecnico di Milano. His interests are in the design and modeling of electrical machines and power electronics. 